\section{Scientists are well placed to speak up for biodiversity}

SIR - As reported in your News story

"Should conservation biologists push policies?" (Nature 442, 13; 2006), academics are reluctant to demand the implementation of schemes to halt and reverse the biodiversity crisis. Conservation scientists seem to fear that taking a position would mean appearing biased, and that this would be better left to others.

Using science to conserve biodiversity does not require a biased opinion: it simply requires belief in the results. There is ample evidence showing that ecosystem, species and genetic diversity are critical for mankind's well-being, and that biodiversity is dedining.

Conservation scientists should follow the path of Richard Doll, who revealed that smoking causes lung cancer (R. Doll and A. B. Hill. Br. Med. J. 2, 739-748; 1950) and then became active in advocating changes in public-health policies - without undermining his academic credibility. If Doll, and later his colleague Richard Peto, had adopted a 'back-seat' attitude and not lobbied against the tobacco industry, many more lives would be claimed by lung cancer today than is the case.

Guillaume Chapron

Carnivoreconservation.org

address supplied

\section{Authors were clear about hockey-stick uncertainties}

SIR - Your News story "Academy affirms hockey-stick graph" (Nature 441, 1032;2006) states that the US National Academy of Sciences (NAS) panel "concluded that systematic uncertainties in climate records from before 1600 were not communicated as clearly as they could have been". This conclusion is not stated in the NAS report itself, but formed part of the remarks made by Gerald North, the NAS committee chair, at the press conference announcing the report.

The name of our paper is "Northern Hemisphere temperatures during the past millennium: inferences, uncertainties, and limitations" (Geophys. Res. Lett. 26, 759-762; 1999). In the abstract, we state: "We focus not just on the reconstructions, but on the uncertainties therein, and important caveats ${ }^{n}$ and note that "expanded uncertainties prevent decisive conclusions for the period prior to $\mathrm{AD} 1400^{\circ}$. We conclude by stating: "more widespread high-resolution data are needed before more confident conclusions can be reached. It is hard to imagine how much more explicit we could have been about the uncertainties in the reconstruction; indeed, that was the point of the artide!
The subsequent confusion about uncertainties was the result of poor communication by others, who used our temperature reconstruction without the reservations that we had stated clearly. Raymond S. Bradley*, Malcolm K. Hughest, MichaelE. Mann

*Department of Geosciences,

University of Massachusetts, Amherst,

Massachusetts 01003, USA

†Laboratory of Tree-Ring Research,

University of Arizona, Tucson,

Arizona 85721, USA

†Department of Meteorology,

Pennsylvania State University,

University Park, Pennsylvania 16802, USA

\section{Systems biology could help us harness useful microbes}

SIR - I would like to add my strong support to your Editorial "Infection biology" (Nature $441,255-256$; 2006) praising the innovative approaches to infection biology described in the US National Academies report Treating Infectious Diseases in a Microbial World. We need to move on from viewing infection as the rather exotic province of a handful of specialized 'virulence genes' carried by a small group of pathogenic organisms, to an appreciation of our true complicity in the microbial world.

The initial encounter of a pathogen with the host immune system involves a bewilderingly complex interchange of signals between the two organisms that sets in train a multiplicity of respective adaptive responses. In addition to immune cells and the pathogen itself, our personal complement of $1.5 \mathrm{~kg}$ of intestinal microflora is likely to be a major arbiter in this encounter. Commensal microbes are important in maintaining the networks regulating the innate immune response: they are certainly important in distributing genes mediating resistance to antibiotics, and they may also regulate the way in which we metabolize treatment drugs.

Systems biology provides a framework in which to address this complexity. Moving towards the rational design of novel antimicrobials and novel vaccines particularly novel antimicrobials and vaccines that can be applied in synergistic combinations - involves digestion of huge amounts of information generated by increasingly high-throughput approaches to microbiology, immunology, genetics and population biology. To make sense of this requires a combination of traditional biology skills with the expertise of mathematicians and computer scientists.

In addition to improving our weapons in the 'war' against selected pathogens, a deeper understanding of the ways in which we interact with microbes is crucial in combating a wide range of diseases and disorders. The population of microbes that has a vested interest in a healthy human population is very much larger than that which seeks our destruction. Systems biology may help us turn this fact to our advantage. Douglas Young

Centre for Integrative Systems Biology, Imperial College London, Flowers Building, Armstrong Road, London SW7 2AZ, UK wnw. imperial.ac.uk/cisbic

\section{Sticking points in the push for change}

SIR — Your Editorial " Reaching a tipping point ${ }^{\text {" }}$ (Nature 441,$785 ; 2006$ ) discusses in the context of climate change the concept of a 'tipping point,' from Malcolm Gladwell's book of the same name: that extraordinary change is possible if only a few key pre-conditions come together appropriately.

Gladwell's proposition embeds an older, specious belief that directed change of the social or political world is easy, perhaps even easier than changing the physical world. But compare, for example, the building of the first atomic bomb with the elimination of racism or poverty.

When social change does occur, it is seldom as dramatic as the kinds of change described by tipping points. There is considerable continuity in social systems because of the resilience of institutions, and because, as articulated by Anthony Giddens in Modernity and Self-Identity: Self and Society in the Late Modern Age (Stanford Univ. Press, Stanford, 1991), of the basic human need for 'ontological security' - a firm belief in the continuity of social and material environments, and in the endurance of social patterns and individual self-identity.

Even if there were widespread agreement on the need for a specific social change, via a tipping point, the procedures for accomplishing this are entirely unclear. Gladwell writes about the considerable parallels between the idea of tipping points and the diffusion of innovations, but neglects the fact that more than $90 \%$ of innovations fail.

As quoted in your Editorial, Gladwell's argument runs: 'Look at the world around you. It may seem like an immovable, implacable place. It is not. With the slightest push - in just the right place - it can be tipped."

The conclusion is valid, as far as it goes, but is highly misleading because of its rarity. The usual reality is a generally immovable world where a lot of pushing has little effect. Eugene A. Rosa

Thomas S. Foley Institute for Public Policy and Public Service, Washington State University, Pullman, Washington 99164-4020, USA 